\title{
The Parafascicular Thalamic Nucleus Concomitantly Influences Behavioral Flexibility and Dorsomedial Striatal Acetylcholine Output in Rats
}

\author{
Holden D. Brown, ${ }^{1,3}$ Phillip M. Baker, ${ }^{1,3}$ and Michael E. Ragozzino ${ }^{1,2,3}$ \\ ${ }^{1}$ Program in Neuroscience, ${ }^{2}$ Department of Psychology, and ${ }^{3}$ Laboratory of Integrative Neuroscience, University of Illinois at Chicago, Chicago, \\ Illinois 60607
}

Recent evidence suggests that a circuit involving the centromedian-parafascicular (Pf) thalamus and basal ganglia is critical for a shift away from biased actions. In particular, excitatory input from the Pf onto striatal cholinergic neurons may facilitate behavioral flexibility. Accumulating evidence indicates that an endogenous increase in dorsomedial striatal acetylcholine (ACh) output enhances behavioral flexibility. The present experiments investigated whether the rat (Rattus norvegicus) Pf supports flexibility during reversal learning, in part, by modifying dorsomedial striatal ACh output. This was determined first by examining the effects of Pf inactivation, through infusion of the GABA agonists baclofen and muscimol, on place acquisition and reversal learning. Additional experiments examined Pf inactivation on dorsomedial striatal ACh output during reversal learning and a resting condition. Behavioral testing was performed in a cross-maze. In vivo microdialysis combined with HPLC/electrochemical detection was used to sample ACh from the dorsomedial striatum. Pf inactivation selectively impaired reversal learning in a dose-dependent manner. A subsequent study showed that an increase in dorsomedial striatal ACh efflux ( 30\% above basal levels) during reversal learning was blocked by Pf inactivation, which concomitantly impaired reversal learning. In the resting condition, a dose of baclofen and muscimol that blocked a behaviorally induced increase in dorsomedial striatal ACh output did not reduce basal ACh efflux. Together, the present findings indicate that the Pf is an intralaminar thalamic nucleus critical for behavioral flexibility, in part, by directly affecting striatal ACh output under conditions that require a shift in choice patterns.

\section{Introduction}

There is considerable evidence that the intralaminar thalamic nuclei are important for attention and action selection (Burk and Mair, 1998; Bailey and Mair, 2005; Newman and Mair, 2007). The parafascicular thalamic nucleus (Pf) is part of the centromedian (CM)-Pf complex, located in the caudal region of the intralaminar thalamus. This thalamic region has been traditionally proposed to support pain processing (Vogt et al., 2008) or arousal (Steriade and Deschenes, 1984). More recent experiments suggest that the primate $\mathrm{CM}-\mathrm{Pf}$ is critical for processing information that signals a shift in response patterns. For example, CM-Pf neurons exhibit correlated firing to unexpected stimuli or stimuli requiring an unpredicted response (Minamimoto and Kimura, 2002). Because the Pf has reciprocal connections with the basal ganglia (Berendse and Groenewegen, 1990), a region important for response selection (Graybiel, 1998; Balleine et al., 2009; Kimchi and Laubach, 2009), the Pf may relay information to the basal

Received April 27, 2010; revised July 20, 2010; accepted Aug. 9, 2010.

This research was supported by National Institutes of Health Grants NSO43283, AG027951, and P5O HD055751 (M.E.R.), and T32 MH067631 (H.D.B.). We appreciate the assistance of Rachel Hilbert and Lauren Madonna with behavioral testing.

Correspondence should be addressed to Michael E. Ragozzino, Department of Psychology, University of Illinois at Chicago, 1007 West Harrison, Chicago, IL 60607. E-mail: mrago@uic.edu.

DOI:10.1523/JNEUROSCI.2167-10.2010

Copyright $\odot 2010$ the authors $\quad$ 0270-6474/10/3014390-09\$15.00/0 ganglia to signal a change in choice selection for execution of an appropriate strategy.

The Pf sends excitatory projections onto striatal medium spiny neurons and cholinergic interneurons (Wilson et al., 1983; Dubé et al., 1988; Lapper and Bolam, 1992). Electrical stimulation of the primate CM alters firing patterns of striatal neurons, including putative cholinergic interneurons (Nanda et al., 2009). The rat Pf projections to the striatum may be particularly critical for facilitating a shift in choice patterns when environmental contingencies change. In rats, the dorsomedial striatum is critical when conditions require inhibition of one learned choice pattern and shift to a new or different choice pattern (Pisa and Cyr, 1990, Ragozzino et al., 2002a,b, 2004, 2009; Nicolle and Baxter, 2003; O'Neill and Brown, 2007). This type of behavioral flexibility is required in reversal learning tests. Furthermore, dorsomedial striatal acetylcholine (ACh) efflux selectively increases during reversal learning, whereas blockade of the ACh increase impairs reversal learning (Palencia and Ragozzino, 2006; Ragozzino et al., 2009). One possibility, therefore, is that excitatory input from the Pf stimulates cholinergic neuronal activity in the dorsomedial striatum to facilitate behavioral flexibility.

Consistent with anatomical evidence that the Pf provides excitatory input to striatal cholinergic interneurons, Consolo et al. (1996) found that, in freely moving rats, electrical stimulation of the Pf increases ACh efflux in the dorsal striatum. This effect on striatal ACh output is eliminated by the NMDA antagonist AP-5. 
Moreover, infusion of AP-5 into the dorsomedial striatum concomitantly impairs reversal learning and blocks the performanceassociated increase in dorsomedial striatal ACh output (Palencia and Ragozzino, 2006). Thus, the Pf, along with the dorsomedial striatum, may be part of a larger neural system that supports behavioral flexibility. The role of the Pf in behavioral flexibility, however, remains unknown. The purpose of the present studies was to determine whether the Pf is involved in behavioral flexibility, in part, by modifying dorsomedial striatal ACh output. This was investigated by examining the effects of Pf inactivation on acquisition and reversal learning of a place discrimination, as well as on dorsomedial striatal ACh release during reversal learning and a resting condition.

\section{Materials and Methods}

Subjects. The subjects were adult, male Long-Evans rats weighing between 300 and $350 \mathrm{~g}$ at the time of testing. Animals were individually housed in plastic cages $(26.5 \times 50 \times 20 \mathrm{~cm})$ in a temperature $\left(22^{\circ} \mathrm{C}\right)$ and humidity (30\%) controlled environment and placed on a $12 \mathrm{~h}$ light/dark cycle (lights on at 7:00 A.M.). Animals were food restricted to $85-90 \%$ of their ad libitum body weight during the experiment, and water was available ad libitum. Animal care and use was in accordance with the National Institutes for Health Guide for the Care and Use of Laboratory Animals and approved by the University of Illinois Institutional Laboratory Animal Care and Use Committee.

Apparatus. Training and testing occurred in a four-arm cross maze made of black acrylic. Maze arms contained a base that was $10 \mathrm{~cm}$ wide $\times$ $55 \mathrm{~cm}$ long, two side walls that were $15 \mathrm{~cm}$ high $\times 55 \mathrm{~cm}$ long, and a back wall that was $8 \mathrm{~cm}$ wide $\times 15 \mathrm{~cm}$ high. A $10 \times 10 \mathrm{~cm}$ square base piece connected all four arms together. A circular food well $(3.2 \mathrm{~cm}$ diameter and $1.6 \mathrm{~cm}$ deep) was located $3 \mathrm{~cm}$ away from the end of each arm. The maze was elevated $72 \mathrm{~cm}$ above the floor in a room with various extramaze cues. Between trials, rats were placed in a holding area, either on their cage top (experiment 1 ) or in a clear acrylic bowl (experiments $2 \mathrm{~A}$ and $2 \mathrm{~B}$ ).

Surgery. In all experiments, a rat underwent stereotaxic surgery for implantation of bilateral guide cannulae aimed at the Pf. In experiments $2 \mathrm{~A}$ and $2 \mathrm{~B}$, each rat also received bilateral implantation of microdialysis guide cannulae into the dorsomedial striatum. For surgery, rats received $0.2 \mathrm{ml}$ of atropine sulfate $(250 \mu \mathrm{g} / \mathrm{ml}$ solution $) 10$ min before injection of sodium pentobarbital $(50 \mathrm{mg} / \mathrm{kg}$, i.p.). For implantation of guide cannulae into the Pf, 22 gauge stainless steel guide cannulae (Plastics One) were implanted bilaterally into the $\mathrm{Pf}$ at a $15^{\circ}$ angle. The stereotaxic coordinates were as follows: anterior-posterior (AP), $-4.2 \mathrm{~mm}$; medial-lateral (ML), $\pm 2.9 \mathrm{~mm}$; dorsal-ventral (DV), $-5.9 \mathrm{~mm}$. Guide cannulae for the microdialysis probes (CMA Microdialysis) were implanted bilaterally into the dorsomedial striatum at a $10^{\circ}$ angle. The stereotaxic coordinates were as follows: AP, $+0.6 \mathrm{~mm}$; ML, $\pm 2.8 \mathrm{~mm}$; DV, $-3.3 \mathrm{~mm}$. The coordinates were based on the stereotaxic atlas by Paxinos and Watson (1998). Four jeweler screws were positioned in the skull surrounding the cannulae and secured with dental acrylic (Stoetling). Stylets were placed into the guide cannulae to prevent clogging. For experiments $2 \mathrm{~A}$ and $2 \mathrm{~B}$, an omega-shaped ring was implanted on top of the skull posterior to the Pf guide cannulae. This was used to connect to the liquid swivel for the in vivo microdialysis procedure to prevent the lines from becoming twisted. Rats were allowed to recover for $7 \mathrm{~d}$ after surgery. For $5 \mathrm{~d}$ after surgery, rats were fed ad libitum and subsequently food restricted as described above. During this period, subjects were handled $\sim 10 \mathrm{~min} / \mathrm{d}$.

Microinfusion procedure. Infusions into the Pf were delivered by 28 gauge injection cannulae that extended $1 \mathrm{~mm}$ below the guide cannulae. The injection cannulae were connected by polyethylene tubing to a $10 \mu \mathrm{l}$ syringe (Hamilton Company). Saline or a mixture of baclofen (Bac) and muscimol (Mus) (Sigma-Aldrich) was infused bilaterally at a rate $0.15 \mu \mathrm{l}$ over $1.5 \mathrm{~min}$ by a microinfusion pump (74900 Series; Cole Palmer). The total volume injected was $0.15 \mu \mathrm{l}$ in each hemisphere. Injection cannulae were left in place for $1 \mathrm{~min}$ after injection to allow for diffusion. Rats remained in their home cages or acrylic bowl for an additional $5 \mathrm{~min}$ before testing began.
In vivo microdialysis. In experiments $2 \mathrm{~A}$ and $2 \mathrm{~B}$, an in vivo microdialysis procedure was performed. Each rat was placed in an acrylic bowl. By way of the omega-shaped ring attached to the dental cement, a rat was connected to the tether and a $2 \mathrm{~mm}$ microdialysis probe was inserted through either the left or right guide cannula into the dorsomedial striatum. The hemisphere in which the probe was placed was chosen pseudorandomly, such that collection was conducted approximately an equal number of times from each hemisphere within a treatment group. Artificial CSF (aCSF) was perfused through the microdialysis probes at a rate of $2.0 \mu \mathrm{l} / \mathrm{min}$. The aCSF contained the following (in M): $0.01758 \mathrm{NaCl}$, $0.004 \mathrm{KCl}, 0.002 \mathrm{Na}_{2} \mathrm{HPO}_{4}, 0.0006 \mathrm{NaH}_{2} \mathrm{PO}_{4}, 0.001 \mathrm{CaCl}_{2}, 0.0009$ $\mathrm{MgCl}_{2}$, and 0.001 glucose, $\mathrm{pH}$ 7.4. To reliably detect ACh levels, the reversible acetylcholinesterase inhibitor neostigmine bromide (100 nM) was added to the aCSF. The perfusate collected during the first $60 \mathrm{~min}$ was not analyzed to allow for equilibration at the probe site.

ACh assay. Samples $(10 \mu \mathrm{l})$ were assayed for ACh using HPLC with electrochemical detection. Samples were loaded on a microbore analytical column for separation of ACh and choline. After separation, an enzymatic postcolumn reactor containing acetylcholinesterase and choline oxidase converted ACh to choline and acetate and choline to betaine and hydrogen peroxide, respectively. Stoichiometric quantities of hydrogen peroxide were produced from the breakdown of ACh and choline. The hydrogen peroxide was further broken down and detected by a glassy carbon-wired electrode coated with peroxidase operated at $+100 \mathrm{mV}$ versus an $\mathrm{Ag} / \mathrm{AgCl}$ reference electrode (Huang et al., 1995). The mobile phase containing $50 \mathrm{~mm} \mathrm{NaH}_{2} \mathrm{PO}_{4}, 0.3 \mathrm{~mm}$ EDTA, and $0.005 \%$ ProClin (to prevent bacterial growth) was delivered at a rate of $100 \mu \mathrm{l} / \mathrm{min}$ by a solvent delivery system. ACh peaks were quantified by comparison with peak heights of ACh standard solutions. The detection limit was $10 \mathrm{fmol}$, and the assay was completed in $14 \mathrm{~min}$.

Experiment 1: the effects of combined baclofen and muscimol infusion into the Pf on acquisition and reversal learning of a place discrimination. Rats were tested on acquisition and reversal learning of a place discrimination across 2 consecutive days. The same two start arms were used on both acquisition and reversal learning. The start arms were located directly across from each other. Rats were started pseudorandomly from the two different arms such that any start arm was not used for more than two consecutive trials. A black plastic block was placed in the entrance of the maze arm opposite to that of the start arm, giving the maze a T-shape. Thus, the same two choice arms were available no matter what start arm was used. Because the start arms were opposite of each other, a rat had to make opposite turn patterns from each start arm to accurately enter the maze arm containing the cereal reinforcement. Therefore, a rat could not use a response strategy, e.g., always turn right, to learn the task. Previous experiments using probe trials, e.g., transferring rats to a new maze environment or removing extramaze cues, indicate that rats use a place strategy in both acquisition and reversal learning to guide their choice pattern with this procedure (Ragozzino et al., 1999). A rat was placed in the stem arm with only one of the two choice arms baited. In the acquisition phase, one choice arm was designated the reinforced arm. This arm contained a half piece of cereal reinforcement during each trial. In this phase, a rat was required to enter the reinforced arm. If a rat chose the correct arm, the trial was terminated after a rat consumed the cereal piece. If a rat chose the incorrect arm, the trial was terminated after a rat reached the unbaited food well. Between trials, a rat was placed on top of their home cage, which was placed on a table next to the maze. The maze was then wiped down and rebaited if necessary. The intertrial interval was $\sim 15 \mathrm{~s}$. To minimize the use of intramaze cues, the maze was rotated $90^{\circ}$ every fourth trial. The criterion for acquisition of the place discrimination was 10 consecutive correct trials.

On the second day of testing (reversal learning phase), a rat was required to enter the arm opposite to the arm reinforced in acquisition. The same start arms and choice arms were used as in acquisition, but the choice arm not reinforced on acquisition was now reinforced in reversal learning. Criterion for reversal learning was also 10 consecutive correct trials. Additional measures, including perseverative and regressive errors, were analyzed for the reversal learning session as in previous studies (Ragozzino et al., 2002a,b, 2003, 2004). To distinguish between error types, reversal trials were grouped into blocks of four trials. Perseverative 
errors were defined as the number of errors that occurred when a rat obtained at least three of four incorrect responses in the initial trial blocks. Once a rat obtained two of four correct responses in a block, subsequent errors were defined as regressive errors.

Five minutes before each test session, rats were infused with either saline or a micromolar concentration of Bac-Mus according to the procedure described above. Selected doses were similar to those used in other experiments (Clayton and Williams, 2000; Floresco et al., 2006; Murschall and Hauber, 2006). Group assignment was determined by the treatment administered during each test phase (acquisition/reversal learning). The amount of baclofen and muscimol injected is represented in nanomoles. The final sample size for each group is in parentheses: (1) saline/saline $(n=11) ;(2)$ saline/Bac at $0.005-$ Mus at $0.018(n=8) ;(3)$ saline/Bac at $0.05-$ Mus at $0.18(n=8) ;(4)$ saline/Bac at $0.15-$ Mus at 0.53 $(n=10)$; (5) Bac at $0.05-$ Mus at $0.18 /$ saline $(n=7)$; and (6) Bac at $0.15-$ Mus at 0.53 /saline $(n=3)$. Group 1 served as the control group. Groups 2-4 determined whether various doses of Bac-Mus affected reversal learning. Groups 5 and 6 determined whether various doses of Bac-Mus affected acquisition. Thus, different dose combinations of BacMus were administered during acquisition or reversal learning. In subsequent sections, Bac at $0.005-$ Mus at 0.018 is referred to as Bac-Mus low dose, Bac at 0.05-Mus at 0.18 is referred to as Bac-Mus middle dose, and $\mathrm{Bac}$ at $0.15-$ Mus at 0.53 is referred to as Bac-Mus high dose.

Experiment 2A: the effect of combined baclofen and muscimol into the Pf on place reversal learning and simultaneous assessment of dorsomedial striatal ACh output. The goal of experiment $2 \mathrm{~A}$ was to determine whether $\mathrm{Pf}$ inactivation impaired place reversal learning, in part, by blocking a behaviorally induced increase in dorsomedial striatal ACh output. The training procedure was similar as described in previous experiments (Palencia and Ragozzino, 2006; Ragozzino et al., 2009). As in experiment 1, each rat was tested on place acquisition and reversal learning. The in vivo microdialysis procedure was performed only during reversal learning because an increase in dorsomedial striatal ACh output is observed in reversal learning but not initial learning (Ragozzino and Choi, 2004; Ragozzino et al., 2009).

For place acquisition, a rat was placed in the acrylic bowl $1 \mathrm{~h}$ before behavioral testing. Each rat was connected to the microdialysis tether, but no probe was placed in the guide cannula. To maintain consistency across experiments, each rat had an infusion of saline on acquisition. Because Bac-Mus had no effect on acquisition in experiment 1 , this treatment was not administered during acquisition of experiment $2 \mathrm{~A}$. Each rat was trained on acquisition for 50 trials. In previous in vivo microdialysis experiments, rats were tested for a similar number of trials or amount of time during place acquisition (Ragozzino and Choi, 2004; Palencia and Ragozzino, 2006; Ragozzino et al., 2009).

On the following day in the reversal learning test, each rat was again placed in the acrylic bowl and prepared for the in vivo microdialysis procedure as described previously. After $1 \mathrm{~h}$ of equilibration, five baseline samples and one postinfusion baseline sample were collected every $6 \mathrm{~min}$ and analyzed for ACh content. Infusion into the Pf of saline or Bac-Mus began $4.5 \mathrm{~min}$ into the fifth baseline sample. At the sixth baseline sample, the cannulae were left in place for $1 \mathrm{~min}$ to allow for diffusion. After collection of the sixth baseline sample, a rat was placed in the maze for reversal learning. The reversal learning test lasted for $30 \mathrm{~min}$, allowing the collection of five microdialysis samples every $6 \mathrm{~min}$. The number of trials and percentage correct for each 6 min block of testing was recorded. Based on the results from experiment 1, the Bac-Mus low dose did not impair reversal learning, whereas the Bac-Mus middle dose did impair reversal learning. This experiment sought to determine whether an infusion of Bac-Mus into the Pf would block a behaviorally induced increase in striatal ACh output in a dose-dependent manner. Thus, three treatment groups were included in this experiment. Group assignment was determined by the treatment administered during reversal learning with the final sample size for each group in parentheses: (1) saline $(n=7) ;(2)$ Bac-Mus low dose $(n=9)$; (3) Bac-Mus middle dose $(n=8)$.

Experiment 2B: the effect of Pf inactivation on dorsomedial striatal ACh output in a resting condition. One week after experiment $2 \mathrm{~A}$, a rat was placed into the holding chamber to undergo the microdialysis procedure for a second session. The probe was placed into the opposite hemisphere as that in experiment 2A. A similar procedure was used as described in experiment $2 \mathrm{~A}$, except that a rat remained in the holding chamber for the $30 \mathrm{~min}$ after collection of the six baseline samples. Groups were divided by the treatment administered during the resting condition with the final sample size for each group in parentheses: (1) saline $(n=10)$; (2) BacMus middle dose $(n=7)$. Rats that were injected with saline on reversal learning were given Bac-Mus in the resting condition, and rats that received Bac-Mus in reversal learning were given saline in the resting condition. All rats in experiment $2 \mathrm{~B}$ had previously experienced the reversal learning paradigm in experiment $2 \mathrm{~A}$.

Histology. After completion of behavioral testing (experiment 1) or the resting condition (experiment 2), rats were given an overdose of sodium pentobarbital, and $2.5 \%$ Chicago Sky Blue stain was injected into the guide cannulae to help visualize injector tip locations. Rats were intracardially perfused with $0.9 \%$ PBS, followed by $4 \%$ formaldehyde solution. The brain was removed and stored in formaldehyde until sectioning. Brains were frozen and cut into $50 \mu \mathrm{m}$ coronal sections on a cryostat. Sections were immediately mounted on slides, dried, and then stained with cresyl violet. Placements were then verified with reference to the stereotaxic atlas of Paxinos and Watson (1998).

Statistical analysis. In experiment 1 , a one-way ANOVA was performed to determine differences in the number of trials to criterion on both acquisition and reversal learning. A separate ANOVA was performed for the acquisition and reversal learning tests. A one-way ANOVA was also conducted to evaluate differences among the groups on both perseverative and regressive errors. A significant treatment effect was followed by Newman-Keuls post hoc tests to determine significant differences between treatment groups.

In experiments $2 \mathrm{~A}$ and $2 \mathrm{~B}$, the microdialysis data were analyzed by converting the raw values to percentages based on each subject's baseline output. The baseline output was calculated from the mean of the five samples before the infusion for each subject. In experiment $2 \mathrm{~A}$, a repeated-measures ANOVA was used to analyze percentage correct scores across blocks of reversal learning among the groups. Dunnett's tests were used for each treatment group to determine changes in ACh output during the reversal learning phase compared with baseline. Bonferroni's $t$ tests were used to further analyze differences between groups on individual test blocks (Keselman et al., 1991).

\section{Results}

Figure 1 shows photomicrographs of typical cannulae placements into the Pf, as well as a microdialysis probe placement into the dorsomedial striatum.

Pf placements were considered accurate if the cannulae tips were bilaterally located -4.16 to $-4.30 \mathrm{~mm}$ posterior to bregma, lateral to the fasciculus retroflexus and dorsal to the ventral posterior parvocellular nucleus. Thus, accurate cannulae placements were located in the lateral Pf. Cannula placements located outside the Pf were found in a variety of regions surrounding the Pf, most commonly in the mediodorsal thalamic nucleus, the prerubral field, and fasciculus retroflexus.

Of the various cannula misplacements, one striking pattern did emerge during behavioral testing. There were several rats $(n=14)$ in experiment 1 that received either the middle or high dose of Bac-Mus with a cannula placement located anterior to the Pf, for example, in the mediodorsal thalamic nucleus, paracentral thalamic nucleus, or superior cerebellar peduncle, which led to a rat displaying a head tilt and a difficulty in consuming the cereal pieces. These rats stopped performing in the maze and therefore were unable to complete the task.

In experiments $2 \mathrm{~A}$ and $2 \mathrm{~B}$, correct microdialysis probe placements in the dorsomedial striatum were found in the anteroposterior plane at the level of the genu of the corpus callosum. Placements ranged from +0.5 to $+1.6 \mathrm{~mm}$ anterior to bregma. Probe placements were located in the dorsomedial sector of the striatum extending below the overlying corpus callosum and dorsal to the nucleus accumbens. Four rats had microdialysis probe placements primarily 
A

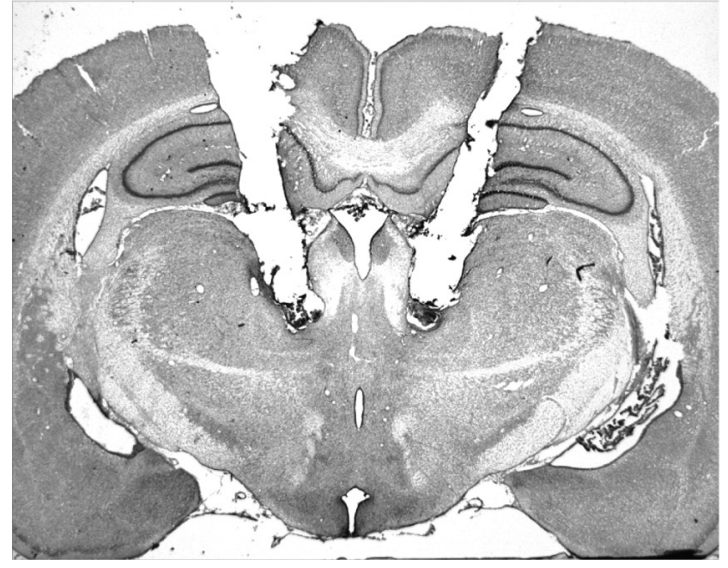

B

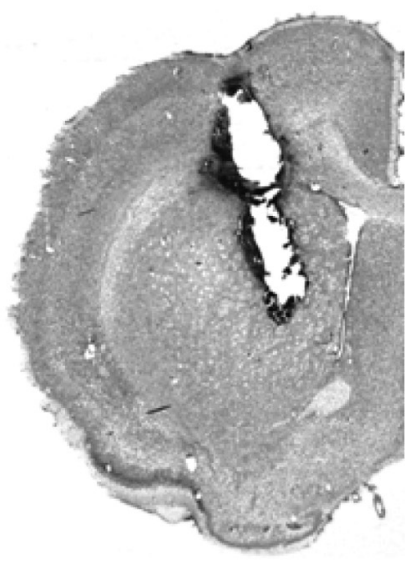

Figure 1. Representative cannula placements in the parafascicular thalamic nucleus and dorsomedial striatum. $\boldsymbol{A}$, Photomicrograph of a typical cannula placement into the parafascicular thalamic nucleus. $\boldsymbol{B}$, Photomicrograph of a typical microdialysis cannula placement into the dorsomedial striatum.

A
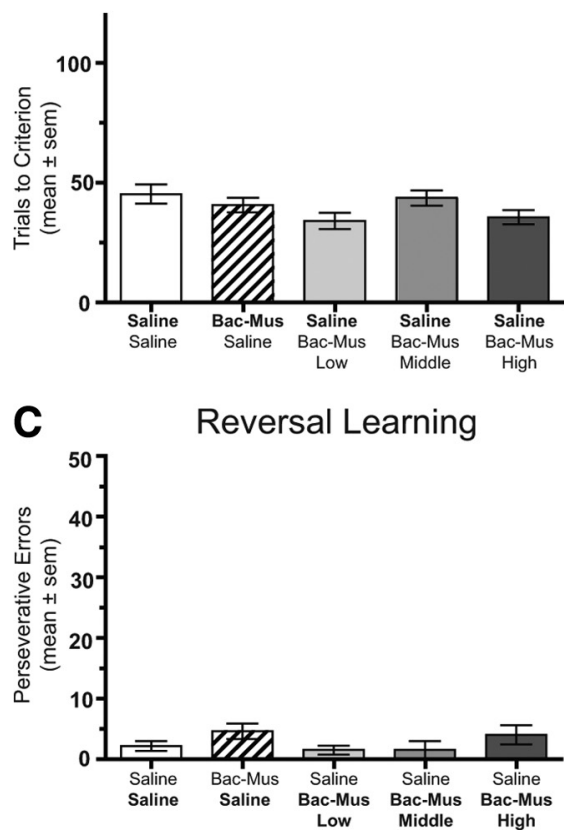

B

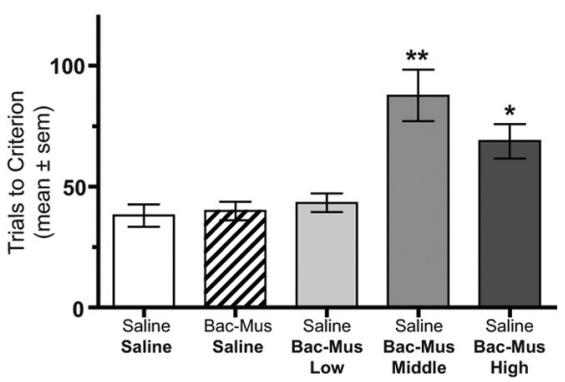

D

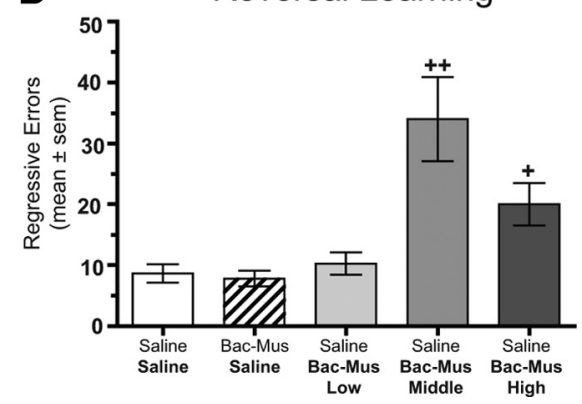

Figure 2. Behavioral performance on the place discrimination in experiment 1. Each rat received bilateral infusions of saline (Sal) or one of three different doses of Bac-Mus into the Pf 5 min before each test session. The treatments on the $x$-axis represent the treatment received before acquisition (top) and before reversal learning (bottom). $\boldsymbol{A}$, Mean $\pm \mathrm{SEM}$ trials to criterion on place acquisition. Infusion of Bac-Mus had no effect on acquisition. $\boldsymbol{B}$, Mean \pm SEM trials to criterion on place reversal learning. The middle and high dose of Bac-Mus significantly impaired reversal learning. ${ }^{*} p<0.05$ versus saline/saline, Bac-Mus/saline, and saline/Bac-Mus low dose. ${ }^{* *} p<0.01$ versus saline/saline, Bac-Mus/saline, and saline/ Bac-Mus low dose. C, Mean \pm SEM perseverative errors committed during reversal learning. Bac-Mus treatment did not affect the number of perseverative errors. $D$, Mean \pm SEM regressive errors committed during reversal learning. The middle and high dose of Bac-Mus significantly increased regressive errors. ${ }^{+} p<0.05$ versus saline/saline, Bac-Mus/saline, and saline/Bac-Mus low dose. ${ }^{++} p<0.01$ versus saline/ saline, Bac-Mus/saline, saline/Bac-Mus low dose, and saline/Bac-Mus high dose.

in the lateral ventricle or in the overlying corpus callosum. The data from these rats were not included in the final analysis.

Experiment 1: the effects of combined baclofen and muscimol infusion into the Pf on acquisition and reversal learning of a place discrimination

The number of trials to reach criterion during acquisition of the place discrimination is illustrated in Figure $2 \mathrm{~A}$. Rats who received an intracranial infusion of either the middle or high dose of Bac-Mus were collapsed into one group because there was no difference between the groups on acquisition. All groups that received a saline infusion before place acquisition required a comparable number of trials to obtain criterion. The means \pm SEM ranged from $34.0 \pm 3.4$ to $45.3 \pm 4.0$. This acquisition rate is comparable with that observed with unoperated rats who have received systemic injections of saline (M. E. Ragozzino, unpublished observations). Inactivation of the Pf did not alter acquisition of the place discrimination compared with that of saline-treated rats. Specifically, inactivation of the Pf with a combination of Bac-Mus resulted in a mean of $40.7 \pm 3.1$ that was comparable with saline controls. An ANOVA indicated that there was not a significant treatment effect on place acquisition $\left(F_{(4,46)}=2.03, p>0.05\right)$. Thus, inactivation of the Pf has no significant effect on acquisition of a place discrimination.

In contrast to place acquisition, BacMus treatment in the Pf significantly impaired reversal learning in a dosedependent manner (Fig. 2B). During reversal learning, saline treated rats required $38.0 \pm 4.6$ trials to achieve criterion. An injection of Bac-Mus low dose into the Pf led to a mean score of $43.3 \pm 3.9$ in reversal learning, comparable with salinetreated controls. However, the trials to criterion in reversal learning increased with an injection of the Bac-Mus middle dose into the Pf (mean of $87.5 \pm 10.7$ ), as well as with an injection of Bac-Mus high dose (mean of $68.7 \pm 7.1$ ). An ANOVA on the trials to criterion during reversal learning revealed that there was a significant treatment effect $\left(F_{(4,46)}=11.53, p<0.01\right)$. Newman-Keuls post hoc tests indicated that the group receiving the Bac-Mus middle dose in reversal learning required significantly more trials to criterion compared with that of all other treatment groups ( $p$ values $<0.01$ ), except the Bac-Mus high dose group in which there was a significant difference between the groups at $p<$ 0.05. Post hoc tests also revealed that the group receiving the Bac-Mus high dose in reversal learning led to significantly greater number of trials to criterion compared with that of the saline or the BacMus low dose groups ( $p$ values $<0.05$ ).

There were five rats that received either the middle or high dose of Bac-Mus in which only one cannula was located in the Pf, whereas the other was located outside the Pf. Unilateral Pf inactivation did not impair reversal learning performance (mean trials to criterion on reversal learning, $45.0 \pm 6.5$ vs saline, $38.0 \pm 4.6$ ). Thus, bilateral Pf inactivation was necessary to impair reversal learning of a place discrimination in a dose-dependent manner. 
There were several rats $(n=13)$ who completed behavioral testing that had bilateral misplacements and received either the middle or high dose of Bac-Mus. Two of these rats had placements that were ventral and extended through the Pf, leading to extensive damage. One rat achieved reversal learning criterion in 70 trials, and the other rat achieved criterion in 71 trials. The other cannula misplacements could be categorized as anterior/ lateral (mediodorsal thalamic nucleus, centrolateral thalamic nucleus, or posterior thalamic nuclear group), posterior (central tegmental tract, superior cerebellar peduncle, or magnocellular nucleus of the posterior commissure), or dorsal (precommissural nucleus or anterior pretectal nucleus) to the Pf. The mean trials to criterion during reversal learning for the groups were as follows: anterior/lateral group $(n=4), 47.3 \pm 4.5 \mathrm{SEM}$; posterior $(n=3)$, $44.3 \pm 4.8$; and dorsal $(n=4), 38.3 \pm 6.7$. Thus, Bac-Mus injections at the middle or high dose in several different thalamic nuclei surrounding the Pf did not produce reversal learning deficits as observed when the same treatments were injected into the Pf.

An analysis of the errors made during reversal learning indicated that all the treatment groups exhibited a similar number of perseverative errors during reversal learning (Fig. 2C). An ANOVA indicated that there was not a significant treatment effect in the number of perseverative errors during reversal learning $\left(F_{(4,46)}=1.36, p>0.05\right)$. However, there was a significant increase in the number of regressive errors among the groups $\left(F_{(4,46)}=10.31, p<0.01\right)$. Specifically, the middle dose of BacMus significantly increased regressive errors compared with that of all other groups ( $p$ values $<0.01$ ). The high dose of Bac-Mus significantly increased regressive errors compared with that of saline controls and the low-dose of Bac-Mus $(p<0.05)$. Thus, inactivation of the Pf thalamus with baclofen and muscimol impairs place reversal learning by selectively increasing regressive errors.

\section{Experiment 2A: the effect of combined baclofen and muscimol into the Pf on place reversal learning and} simultaneous assessment of dorsomedial striatal ACh output The results from Pf inactivation on dorsomedial striatal ACh output during reversal learning are shown in Figure 3. Before infusion, there was no difference in baseline levels of striatal ACh efflux among the treatment groups. After a saline infusion, there was a $26.6 \pm 5.8 \%$ increase in ACh efflux during the first test block (T1) that persisted throughout the reversal session (T2T5), reaching a maximum at T5 $(33.9 \pm 8.1 \%)$. An infusion of the Bac-Mus low dose resulted in a similar increase in dorsomedial striatal ACh output pattern to saline-treated controls. The BacMus low dose showed a $21.4 \pm 2.1 \%$ increase in ACh during the first test block (T1) and reached a maximal increase in T4 (33.9 \pm $4.1 \%)$. In contrast, infusion of the Bac-Mus middle dose into the Pfblocked an increase in dorsomedial striatal ACh output during the entire reversal session (T1-T5) in which a maximal increase occurred in T2, which was only $4.6 \pm 6.5 \%$ above basal output. A repeated-measures ANOVA revealed that there was a significant group effect $\left(F_{(2,18)}=6.87, p<0.01\right)$, reflecting the effect of treatment on dorsomedial striatal ACh efflux. There was a significant effect of block $\left(F_{(9,162)}=32.52, p<0.01\right)$, indicating that ACh output was significantly different across sampling blocks. Furthermore, there was a significant treatment $\times$ block interaction $\left(F_{(18,162)}=4.41, p<0.01\right)$. Dunnett's tests were performed for each treatment group to determine whether dorsomedial striatal ACh output significantly changed in various test blocks compared with baseline. A comparison was made using only the last baseline sample (B5) for each group because all baseline samples

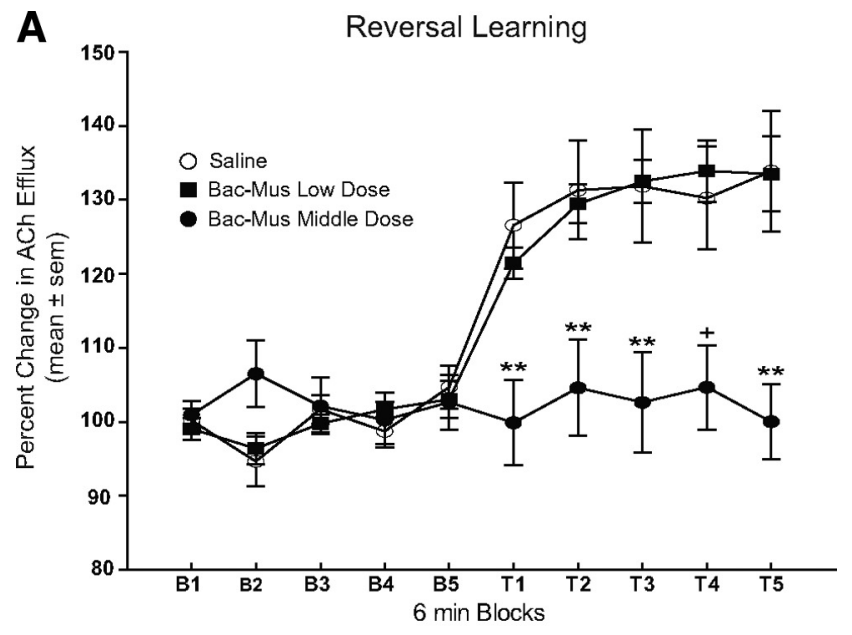

B

Reversal Learning

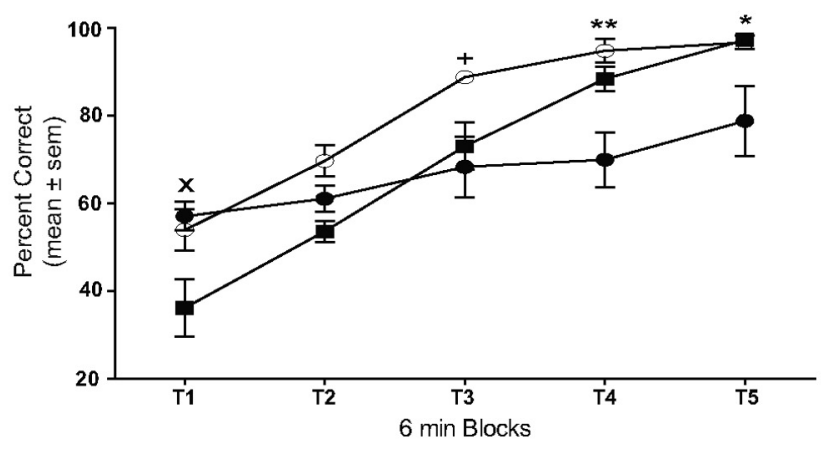

Figure 3. Dorsomedial striatal ACh output and behavioral performance during place reversal learning. Each rat received bilateral infusions of saline (Sal) or one of two different doses of Bac-Mus into the Pf 6 min before reversal learning. Rats were tested for 30 min across five 6-min test blocks (T1-T5). A, ACh output in the dorsomedial striatum during reversal learning. Dorsomedial striatal ACh output significantly increased above basal levels in the saline and the Bac-Mus low-dose groups. The middle dose of Bac-Mus infused into the Pf significantly attenuated the performance-induced increase in dorsomedial striatal $A C h$ efflux throughout reversal learning. ${ }^{+} p<0.05$ versus saline and $p<0.01$ versus $\mathrm{Bac}-$ Mus low dose. ${ }^{* *} p<0.01$ versus saline and Bac-Mus low dose. $\boldsymbol{B}$, Mean percentage correct during place reversal learning. Infusion of the low dose of Bac-Mus into the Pf significantly reduced performance during $\mathrm{T1}$ compared with that of other treatment groups. Infusion of the middle dose of Bac-Mus into the Pf significantly reduced reversal learning performance compared with that of the other treatment groups during $\mathrm{T} 4$ and $\mathrm{T} 5$ and also compared with saline in T3. ${ }^{\mathrm{x}} p<0.05$ saline and Bac-Mus middle dose versus $\mathrm{Bac}-$ Mus low dose. ${ }^{+} p<0.05$ saline versus $\mathrm{Bac}-$ Mus middle dose. ${ }^{*} p<$ $0.05 \mathrm{Bac}-$ Mus middle dose versus saline and $\mathrm{Bac}-$ Mus low dose. ${ }^{* *} p<0.01 \mathrm{Bac}-$ Mus middle dose versus saline and Bac-Mus low dose.

were similar. Dunnett's tests revealed that there was a significant increase in dorsomedial striatal ACh output during all reversal learning blocks compared with basal levels for saline controls ( $p$ values $<0.01$ ) and Bac-Mus low dose ( $p$ values $<0.05$ ). However, treatment with the Bac-Mus middle dose led to ACh output that was not significantly different from that of baseline throughout the reversal learning session ( $p$ values $>0.05$ ).

To further examine the treatment $\times$ block interaction, Bonferroni's $t$ tests were conducted to analyze differences between groups at individual test blocks. There was no significant difference between any of the groups during the baseline periods ( $p$ values $>0.05$ ). The Bac-Mus middle dose exhibited significantly lower dorsomedial striatal ACh output than both saline and BacMus low dose groups in blocks T1-T5 ( $p$ values $<0.01$, except in block T4 vs saline $p<0.05$ ). Thus, administration of the Bac-Mus middle dose into the Pf significantly blocked an increase in dor- 
somedial striatal ACh efflux during reversal learning. In contrast, Bac-Mus low dose exhibited an increase in dorsomedial striatal ACh efflux similar to that of controls during reversal learning ( $p$ values $>0.05$ ).

For the behavioral testing in this experiment, all groups received an infusion of saline into the Pf before place acquisition. The three groups performed at a similar level during the last 10 trials of place acquisition (acquisition/reversal learning: saline/ saline, $95.0 \pm 1.9 \%$; saline/Bac-Mus low dose, $97.0 \pm 1.5 \%$; saline/Bac-Mus middle dose, $91.3 \pm 4.0 \%$ ). A one-way ANOVA indicated that there was not a significant difference in place acquisition performance among the groups during the final 10 trials $\left(F_{(2,23)}=1.32, p>0.05\right)$.

In reversal learning, the Bac-Mus middle dose not only blocked a behaviorally induced increase in dorsomedial striatal ACh output but concomitantly impaired behavioral performance (Fig. 3B). During the initial trials of reversal learning, rats chose the arm that was correct on acquisition but subsequently began to choose the opposite arm that now contained the food reinforcement. In $\mathrm{T} 1$, the Bac-Mus middle dose group had a mean score of $57.1 \pm 3.3 \%$, whereas saline controls had a mean score of $54.0 \pm 4.7 \%$. The Bac-Mus low dose at T1 impaired performance compared with that of the other two groups with a mean score of $36.2 \pm 6.6 \%$. Saline-treated controls and the BacMus low-dose group steadily improved their performance across the reversal learning session such that both groups achieved near perfect performance by the final test block (saline, $96.7 \pm 1.6 \%$; Bac-Mus low dose, $97.3 \pm 1.3 \%)$. The Bac-Mus middle dose group showed a slower rate of learning in the reversal session only achieving $79.0 \pm 8.0 \%$ correct by the final test block. A two-way ANOVA with repeated measures revealed there was a significant treatment effect $\left(F_{(2,81)}=6.61, p<0.01\right)$. There was also a significant block effect $\left(F_{(4,84)}=50.47, p<0.01\right)$. Furthermore, the treatment $\times$ block interaction was significant $\left(F_{(8,84)}=4.81, p<\right.$ $0.01)$. Bonferroni's $t$ tests revealed that the Bac-Mus low dose had significantly lower reversal learning performance in T1 compared with that of saline controls and the Bac-Mus middle dose ( $p$ values $<0.01)$. Additional tests revealed a significant difference in performance between saline controls and Bac-Mus middledose group during blocks T3-T5 ( $p$ values $<0.05$ ). In addition, Bac-Mus middle dose had a significantly lower percentage correct compared with that of Bac-Mus low dose at blocks T4 and T5 ( $p$ values $<0.05$ ). Thus, the Bac-Mus middle dose into the Pf impaired reversal learning similar to that observed in experiment 1.

One possibility is that the Bac-Mus middle dose into the Pfled to hyperactivity or hypoactivity, causing a secondary effect on reversal learning performance. To examine this possibility, the number of trials completed in each block was examined. All groups completed a comparable number of trials across all test blocks, with each group completing an average of $8-10$ trials per block (supplemental Fig. 1, available at www.jneurosci.org as supplemental material). A repeated-measures ANOVA indicated there was not a significant treatment effect $\left(F_{(2,84)}=1.63, p>\right.$ $0.05)$, block effect $\left(F_{(4,84)}=1.49, p>0.05\right)$, or treatment $\times$ block interaction $\left(F_{(8,84)}=1.30, p>0.05\right)$. Therefore, Bac-Mus at the low or middle dose did not have an effect on general activity as measured by the number of trials completed.

There were seven rats in experiment $2 \mathrm{~A}$ that received the middle dose of Bac-Mus into the Pf that were excluded from the analysis because of cannula misplacements. Three of these rats displayed motor impairments as described above. The other four rats had placements that were either rostral or caudal to Pf. In

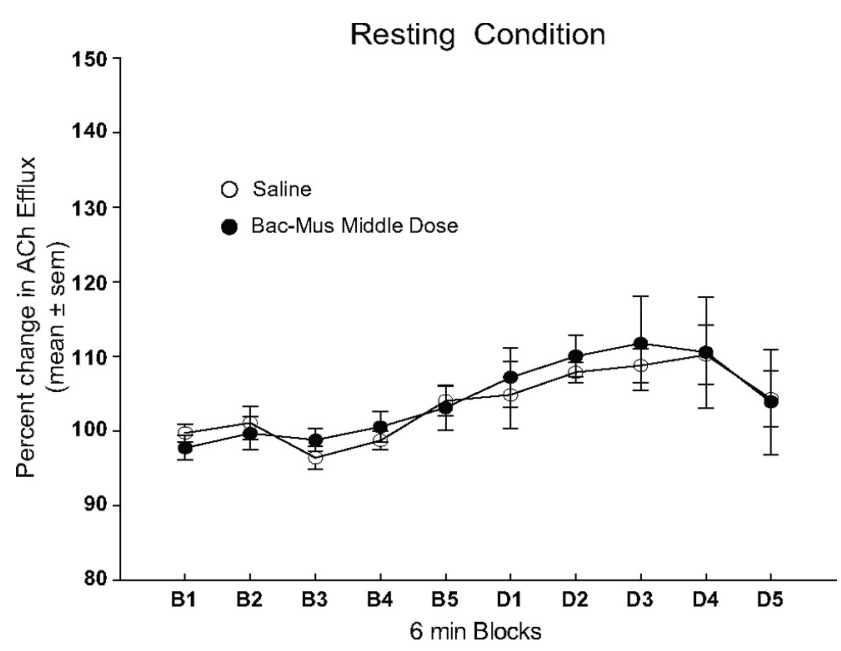

Figure 4. The effect of parafascicular inactivation on ACh output in the dorsomedial striatum during a resting condition. Samples were collected at 6 min intervals. Infusion of either saline or the middle dose of Bac-Mus had no effect on basal ACh efflux.

these four rats, the mean \pm SEM percentage correct during the first test block was $47.7 \pm 0.7$, and the percentage correct in the final test block was $86.7 \pm 14.0$. This performance was comparable with that observed in saline controls. Two of these rats had an accurate microdialysis placement in the dorsomedial striatum. During reversal learning, one rat exhibited a maximal increase in ACh output of $24.8 \%$ above basal levels, and the other rat had a maximal increase in ACh output of $37.4 \%$ above basal levels. Thus, despite receiving the middle dose of Bac-Mus, when cannula placements were located outside the Pf, there was an increase in dorsomedial striatal ACh output during reversal learning comparable with that observed in saline controls.

\section{Experiment 2B: the effect of combined baclofen and muscimol into the Pf on dorsomedial striatal ACh output during a resting condition}

The findings from experiment $2 \mathrm{~A}$ revealed that the Bac-Mus middle dose simultaneously impaired place reversal learning and blocked a behaviorally induced increase in dorsomedial striatal ACh output. To determine whether this dose of Bac-Mus altered basal ACh output, experiment $2 \mathrm{~B}$ investigated the effect of infusing the Bac-Mus middle dose in the Pf on striatal ACh output in a resting condition. As shown in Figure 4, the Bac-Mus middle dose did not alter basal ACh output from the dorsomedial striatum compared with that of saline-treated controls. ACh efflux in both the saline and Bac-Mus middle dose groups slightly increased ( $\sim 10 \%$ above preinjection levels) over time. A repeatedmeasures ANOVA revealed a significant effect of block $\left(F_{(9,117)}=\right.$ 4.57, $p<0.01)$. However, there was not a significant treatment effect $\left(F_{(1,13)}=0.18, p>0.05\right)$ or treatment $\times$ block interaction $\left(F_{(9,117)}=0.87, p>0.05\right)$.

\section{Discussion}

The present experiments demonstrated that the Pf interacts with a prime neuromodulatory system in the striatum, ACh, to support behavioral flexibility. Pf inactivation did not affect place acquisition but impaired place reversal learning. The reversal learning deficit observed after Pf inactivation is consistent with results indicating that posterior intralaminar thalamic lesions, which include the Pf, do not impair acquisition of various discrimination tests (Nyakas et al., 1985; Savage et al., 1997; QuirozPadilla et al., 2007) but do impair reversal learning (Thompson et 
al., 1981; Nyakas et al., 1985). In past experiments, lesions extended beyond the Pf and damaged fibers of passage. The current study pharmacologically targeted the Pf to temporarily inactivate this region. In contrast to Pf inactivation, the middle and high doses of Bac-Mus injected into several adjacent thalamic subregions did not impair reversal learning. Furthermore, different from bilateral Pf inactivation, unilateral Pf inactivation did not impair reversal learning. This suggests that bilateral Pf inactivation is necessary to observe a reversal learning deficit. Importantly, unlike past studies, experiment 1 provides a clear demonstration that specific manipulation of the Pf within the intralaminar thalamic nuclei is sufficient to modify a flexible shift of choice patterns.

Although inactivating adjacent thalamic sites did not affect reversal learning, the effects of Pf inactivation on reversal learning are comparable with studies by demonstrating that central thalamic subregion lesions, located rostral to the Pf, impair switching response patterns (Bailey and Mair, 2005; Newman and Mair, 2007). In contrast to Pf inactivation, injections of GABA agonists into the mediodorsal thalamus did not impair reversal learning, consistent with studies showing that mediodorsal thalamic lesions do not impair reversal learning or only impair performance in serial reversal learning (Beracochea et al., 1989; Neave et al., 1993; Chudasama et al., 2001). Manipulations of the mediodorsal thalamus do affect strategy switching (Block et al., 2007). The mediodorsal thalamus has reciprocal connections with the medial prefrontal cortex, an area critical for strategy switching (Ragozzino et al., 2003; Block et al., 2007), and thus this circuit may be critical when conditions require a shift in strategies but not reversal learning.

The reversal learning deficit induced by Pf inactivation did not impair the ability to initially inhibit the previously correct choice pattern (perseverative errors) but selectively impaired maintaining a new correct choice pattern once selected (regressive errors). In both experiments, Bac-Mus-treated rats initially chose the previously correct spatial location but began to choose the new correct location within a few trials similar to that of controls. The Bac-Mus-treated rats, at the middle and high dose, although able to initially shift away from the previously correct choice pattern were unable to reliably execute the new choice pattern as effectively as controls. Thus, Pf inactivation impaired learning a new choice pattern after initially selected.

The selective increase in regressive errors after Pf inactivation is the same error pattern observed after dorsomedial striatal inactivation (Ragozzino et al., 2002; Ragozzino and Choi, 2004). The similar error pattern produced by Pf or dorsomedial striatum manipulations suggests that the Pf input to the dorsomedial striatum is particularly important for behavioral flexibility. This is supported by the findings showing that Pf inactivation concomitantly blocks a behaviorally induced increase in dorsomedial striatal ACh output and impairs reversal learning. Previous experiments demonstrated that an increase in dorsomedial ACh output is important for a flexible shift in choice patterns (Ragozzino and Choi, 2004; Palencia and Ragozzino, 2006; Ragozzino et al., 2009). The Pf has direct projections to striatal cholinergic interneurons (Lapper and Bolam, 1992). Furthermore, CM-Pf inactivation alters task-dependent firing of striatal tonically active neurons, many of which are presumed to be cholinergic interneurons (Matsumoto et al., 2001). The present experiments extend the anatomical and electrophysiological findings on thalamo-striatal interactions by demonstrating that rat Pf inactivation simultaneously blocks a behaviorally induced increase in striatal ACh output and impairs behavioral flexibility.
This indicates that thalamo-striatal circuitry plays an important role in executing a switch in choice patterns.

An alternative interpretation for Pf inactivation altering striatal ACh output and reversal learning is that adjacent thalamic subregions were principally inactivated. However, Bac-Mus infusions into several thalamic areas bordering the Pf did not impair reversal learning. Furthermore, stimulation of thalamic subregions and fiber tracts bordering the Pf do not affect striatal ACh output, in contrast to direct stimulation of the Pf (Baldi et al., 1995). Another possibility is that Pf inactivation altered striatal ACh output during reversal learning indirectly, by affecting activity in frontal cortex areas that project to the dorsomedial striatum. However, the error pattern observed in reversal learning with Pf inactivation is consistent with a direct modification of dorsomedial striatal activity but not prefrontal cortex activity (Ragozzino, 2007). Second, the Pf does not project to certain frontal cortex areas involved in reversal learning, such as the orbitofrontal cortex (Berendse and Groenewegen, 1991). Third, electrical stimulation of the Pf increases striatal ACh output that is not blocked by deafferentation of the cortical-striatal pathway (Consolo et al., 1996). Therefore, reducing input from medial frontal cortex areas, shown to be involved in reversal learning, e.g., infralimbic and anterior cingulate cortex, does not prevent inhibit Pf stimulation from increasing striatal ACh output. Related to the present findings, the most parsimonious explanation is that Pf inactivation concomitantly produces a reversal learning deficit and blocks an increase in dorsomedial striatal ACh output by disrupting Pf input to striatal cholinergic interneurons.

The Pf projections to the striatum are excitatory (Streit, 1980; Wilson et al., 1983; Dubé et al., 1988), and systemic injection of an NMDA antagonist blocks the Pf stimulation-induced increase in striatal ACh output (Consolo et al., 1996). Consistent with these findings, direct infusion of an NMDA antagonist into the dorsomedial striatum simultaneously impairs reversal learning and blocks an increase in striatal ACh output comparable with that observed with Pf inactivation (Palencia and Ragozzino, 2006). These results suggest that Pf neurons directly activate striatal cholinergic interneurons that are mediated, at least in part, by NMDA receptors when conditions require a shift in choice patterns.

Similar to controls, the Bac-Mus low dose led to an increase in dorsomedial striatal ACh output during reversal learning. In this group, performance was reduced during the first test block compared with saline controls and the middle-dose drug group. Despite this, the Bac-Mus low-dose group exhibited a comparable learning rate as controls and achieved a similar accuracy during the last two test blocks. This contrasts with the middle-dose group that exhibited a reversal learning deficit in the last two test blocks. The Bac-Mus low-dose group exhibited lower performance initially in experiment 2 but did not exhibit this in experiment 1 . One possible explanation for this lower performance is that, unlike experiment 1 , bilateral cannulae were implanted into the dorsomedial striatum leading to some mechanical damage. This may have set up a condition such that a lower dose of baclofen and muscimol into the Pf could produce a small and transient effect on reversal learning.

The present experiments showed through in vivo neurochemical measurements that Pf input to striatal cholinergic interneurons supports behavioral flexibility. Electrophysiological evidence further suggests that this connection is important for behavioral flexibility. Recordings of striatal neurons indicate that this area is critical for facilitating the speed and selection of actions yielding the highest value (Apicella et al., 
1992; Samejima et al., 2005). Specifically, a previous study showed that striatal tonically active neurons have pre-movement activity in a context that requires flexible switching of response patterns (Apicella et al., 1991). In a complementary manner, CM-Pf neurons are activated by alerting unexpected stimuli or when conditions require an action that is unexpected or occurs at a low probability (Minamimoto et al., 2005). Furthermore, CM-Pf inactivation almost completely abolishes the correlated firing pattern in tonically active neurons to behaviorally relevant stimuli (Matsumoto et al., 2001). Because CM-Pf neurons receive input from various sensory modalities and basal ganglia output structures (Sadikot and Rymar, 2009), CM-Pf neurons may be critical for processing stimuli that are previously irrelevant, necessitating selection of a different action to receive a reinforcement, as is the case in reversal learning. In particular, the Pf receives visceral input via specific brainstem regions such as the parabrachial nucleus and nucleus of the solitary tract (Ito and Craig, 2005). One possibility is that, during reversal learning when a certain choice pattern no longer leads to reward, there are certain autonomic changes in which visceral information feeds into the Pf, which then alters ongoing striatal activity switching basal ganglia activity from "top-down" control to "sensorydriven" control (Kimura et al., 2004). Together, these results have led to the proposal that the CM-Pf input to the striatum is critical for reshaping striatal neuronal activity that allows a shift away from a biased choice pattern to selection of a more appropriate choice pattern when environmental contingencies change (Minamimoto et al., 2009). The current experiments provide the first direct demonstration of this proposal, showing that the Pf input to the striatal cholinergic interneurons is critical during a shift in choice patterns.

\section{References}

Apicella P, Scarnati E, Schultz W (1991) Tonically discharging neurons of monkey striatum respond to preparatory and rewarding stimuli. Exp Brain Res 84:672-675.

Apicella P, Scarnati E, Ljungberg T, Schultz W (1992) Neuronal activity in monkey striatum related to the expectation of predictable environmental events. J Neurophysiol 68:945-960.

Bailey KR, Mair RG (2005) Lesions of specific and nonspecific thalamic nuclei affect prefrontal cortex-dependent aspects of spatial working memory. Behav Neurosci 119:410-419.

Baldi G, Russi G, Nannini L, Vezzani A, Consolo S (1995) Trans-synaptic modulation of striatal ACh release in vivo by the parafascicular thalamic nucleus. Eur J Neurosci 7:1117-1120.

Balleine BW, Liljeholm M, Ostlund SB (2009) The integrative function of the basal ganglia in instrumental conditioning. Behav Brain Res 199:43-52.

Beracochea DJ, Jaffard R, Jarrard LE (1989) Effects of anterior or dorsomedial thalamic ibotenic lesions on learning and memory in rats. Behav Neural Biol 51:364-376.

Berendse HW, Groenewegen HJ (1990) Organization of the thalamostriatal projections in the rat, with special emphasis on the ventral striatum. J Comp Neurol 299:187-228.

Berendse HW, Groenewegen HJ (1991) Restricted cortical termination fields of the midline and intralaminar thalamic nuclei in the rat. Neuroscience 42:73-102.

Block AE, Dhanji H, Thompson-Tardif SF, Floresco SB (2007) Thalamicprefrontal cortical-ventral striatal circuitry mediates dissociable components of strategy set shifting. Cereb Cortex 17:1625-1636.

Burk JA, Mair RG (1998) Thalamic amnesia reconsidered: excototoxic lesions of the intralaminar nuclei, but not the mediodorsal nucleus, disrupts place delayed matching-to-sample performance in rats (Rattus norvegicus). Behav Neurosci 112:54-67.

Chudasama Y, Bussey TJ, Muir JL (2001) Effects of selective thalamic and prelimbic cortex lesions on two types of visual discrimination and reversal learning. Eur J Neurosci 14:1009-1020.

Clayton EC, Williams CL (2000) Posttraining inactivation of excitatory af- ferent input to the locus coeruleus impairs retention in an inhibitory avoidance learning task. Neurobiol Learn Mem 73:127-140.

Consolo S, Baldi G, Giorgi S, Nannini L (1996) The cerebral cortex and parafascicular thalamic nucleus facilitate in vivo acetylcholine release in the rat striatum through distinct glutamate receptor subtypes. Eur J Neurosci 8:2702-2710.

Dubé L, Smith AD, Bolam JP (1988) Identification of synaptic terminals of thalamic or cortical origin in contact with distinct medium-size spiny neurons in the rat neostriatum. J Comp Neurol 267:455-471.

Floresco SB, Ghods-Sharifi S, Vexelman C, Magyar O (2006) Dissociable roles for the nucleus accumbens core and shell in regulating set shifting. J Neurosci 26:2449-2457.

Graybiel AM (1998) The basal ganglia and chunking of action repertoires. Neurobiol Learn Mem 70:119-136.

Huang T, Yang L, Gitzen J, Kissinger PT, Vreeke M, Heller A (1995) Detection of basal acetylcholine in rat brain microdialysate. J Chromatogr B Biomed Sci Appl 670:323-327.

Ito S, Craig AD (2005) Vagal-evoked activity in the parafascicular nucleus of the primate thalamus. J Neurophysiol 94:2976-2982.

Keselman HJ, Carriere KC, Shaffer JR (1991) Multiple pairwise comparisons of repeated measures means under violation of multisample sphericity. Psychol Bull 110:162-170.

Kimchi EY, Laubach M (2009) Dynamic encoding of action selection by the medial striatum. J Neurosci 29:3148-3159.

Kimura M, Minamimoto T, Matsumoto N, Hori Y (2004) Monitoring and switching of cortico-basal ganglia loop functions by the thalamo-striatal system. Neurosci Res 48:355-360.

Lapper SR, Bolam JP (1992) Input from the frontal cortex and the parafascicular nucleus to cholinergic interneurons in the dorsal striatum of the rat. Neuroscience 51:533-545.

Matsumoto N, Minamimoto T, Graybiel AM, Kimura M (2001) Neurons in the thalamic CM-Pf complex supply striatal neurons with information about behaviorally significant sensory events. J Neurophysiol 85:960-976.

Minamimoto T, Kimura M (2002) Participation of the thalamic CM-Pf complex in attentional orienting. J Neurophysiol 87:3090-3101.

Minamimoto T, Hori Y, Kimura M (2005) Complimentary process to response bias in the centromedian thalamus. Science 308:1798-1801.

Minamimoto T, Hori Y, Kimura M (2009) Roles of the thalamic CM-PF coupled-basal ganglia circuit in externally driven rebias of action. Brain Res Bull 78:75-79.

Murschall A, Hauber W (2006) Inactivation of the ventral tegmental area abolished the general excitatory influence of Pavlovian cues on instrumental performance. Learn Mem 13:123-126.

Nanda B, Galvan A, Smith Y, Wichmann T (2009) Effects of stimulation of the centromedial nucleus of the thalamus on the activity of striatal cells in awake rhesus monkeys. Eur J Neurosci 29:588-598.

Neave N, Sahgal A, Aggleton JP (1993) Lack of effect of dorsomedial thalamic lesions on automated tests of spatial memory in the rat. Behav Brain Res 55:39-49.

Newman LA, Mair RG (2007) Cholinergic modulation of visuospatial responding in central thalamus. Eur J Neurosci 26:3543-3552.

Nicolle MM, Baxter MG (2003) Glutamate receptor binding in the frontal cortex and dorsal striatum of aged rats with impaired attentional setshifting. Eur J Neurosci 18:3335-3342.

Nyakas C, Veldhuis HD, De Wied D (1985) Beneficial effect of chronic treatment with Org 2766 and -MSH on impaired reversal learning of rats with bilateral lesions of the parafascicular area. Brain Res Bull 15:257-265.

O'Neill M, Brown VJ (2007) The effect of striatal dopamine depletion and the adenosine A2A antagonist KW-6002 on reversal learning in rats. Neurobiol Learn Mem 88:75-81.

Palencia CA, Ragozzino ME (2006) The effect of $N$-methyl-D-aspartate receptor blockade on acetylcholine efflux in the dorsomedial striatum during response reversal learning. Neuroscience 143:671-678.

Paxinos G, Watson C (1998) The rat brain in stereotaxic coordinates, Ed 3. New York: Elsevier.

Pisa M, Cyr J (1990) Regionally selective roles of the rat's striatum in modality-specific discrimination learning and forelimb reaching. Behav Brain Res 37:281-292.

Quiroz-Padilla MF, Guillazo-Blanch G, Vale-Martínez A, Torras-García M, Martí-Nicolovius M (2007) Effects of parafascicular excitotoxic lesions 
on two-way active avoidance and odor discrimination. Neurobiol Learn Mem 88:198-207.

Ragozzino ME (2007) The contribution of the medial prefrontal cortex, orbitofrontal cortex and dorsomedial striatum to behavioral flexibility. Ann N Y Acad Sci 1121:355-375.

Ragozzino ME, Choi D (2004) Dynamic changes in acetylcholine output in the medial striatum during place reversal learning. Learn Mem 11:70-77.

Ragozzino ME, Detrick S, Kesner RP (1999) Involvement of the prelimbicinfralimbic areas of the rodent prefrontal cortex in behavioral flexibility for place and response learning. J Neurosci 19:4585-4594.

Ragozzino ME, Jih J, Tzavos A (2002a) Involvement of the dorsomedial striatum in behavioral flexibility: role of muscarinic cholinergic receptors. Brain Res 953:205-214.

Ragozzino ME, Ragozzino KE, Mizumori SJ, Kesner RP (2002b) Role of the dorsomedial striatum in behavioral flexibility for response and visual cue discrimination learning. Behav Neurosci 116:105-115.

Ragozzino ME, Kim J, Hassert D, Minniti N, Kiang C (2003) The contribution of the rat prelimbic-infralimbic areas to different forms of task switching. Behav Neurosci 117:1054-1065.

Ragozzino ME, Mohler EG, Prior M, Palencia CA, Rozman S (2009) Acetylcholine activity in selective striatal regions supports behavioral flexibility. Neurobiol Learn Mem 91:13-22.
Sadikot AF, Rymar VV (2009) The primate centromedian-parafascicular complex: anatomical organization with a note on neuromodulation. Brain Res Bull 78:122-130.

Samejima K, Ueda Y, Doya K, Kimura M (2005) Representation of actionspecific reward values in the striatum. Science 310:1337-1340.

Savage LM, Sweet AJ, Castillo R, Langlais PJ (1997) The effects of lesions to thalamic lateral internal medullary lamina and posterior nuclei on learning, memory and habituation in the rat. Behav Brain Res 82:133-147.

Steriade M, Deschenes M (1984) The thalamus as a neuronal oscillator. Brain Res 320:1-63.

Streit P (1980) Selective retrograde labeling indicating the transmitter of neuronal pathways. J Comp Neurol 191:429-463.

Thompson R, Kao L, Yang S (1981) Rapid forgetting of individual spatial reversal problems in rats with parafascicular lesions. Behav Neural Biol 33:1-16.

Vogt BA, Hof PR, Friedman DP, Sikes RW, Vogt LJ (2008) Norepinephrinergic afferents and cytology of the macaque monkey midline, mediodorsal, and intralaminar thalamic nuclei. Brain Struct Funct 212:465-479.

Wilson CJ, Chang HT, Kitai ST (1983) Origins of post synaptic potentials evoked in spiny neostriatal projection neurons by thalamic stimulation in the rat. Exp Brain Res 51:217-226. 